\title{
Inclusion Properties of The Homogeneous Herz-Morrey
}

\author{
Hairur Rahman \\ Department of Mathematics, Islamic State University of Maulana Malik Ibrahim Malang \\ Email: hairur@mat.uin-malang.ac.id
}

\begin{abstract}
In this paper, we have discussed the inclusion properties of the homogeneous Herz-Morrey spaces and the homogeneous weak homogeneous spaces. We also studied the inclusion relation between those spaces.
\end{abstract}

Keywords: homogeneous Herz-Morrey spaces; homogeneous weak Herz-Morrey spaces; inclusion properties.

\section{INTRODUCTION}

The subject discussion about inclusion properties of any spaces or inclusion relation between spaces has interested to study. Some authors have studied about inclusion relation in some spaces (see [1], [2], [3], [4] and [5]). It guided us to discuss the inclusion properties of other spaces.

Regarding C.B. Morrey in [6] who introduced Morrey spaces, many authors have defined the generalization of Morrey spaces and combined with other spaces. Lu and Xu [7] introduce one of the homogeneous Herz-Morrey spaces. These spaces are the generalization of Morrey spaces and Herz spaces. Let $\alpha \in \mathbb{R}, 0<p \leq \infty, 0<q<\infty$, and $0 \leq \lambda<\infty$, the homogeneous Herz-Morrey spaces $\mathcal{M} \dot{K}_{p, q}^{\alpha, \lambda}\left(\mathbb{R}^{n}\right)$ are defined by

where

$$
\mathcal{M} \dot{K}_{p, q}^{\alpha, \lambda}\left(R^{n}\right):=\left\{f \in L_{l o c}^{q}\left(\mathbb{R}^{n} /\{0\}\right):\|f\|_{\mathcal{M} \dot{K}_{p, q}^{\alpha, \lambda}\left(\mathbb{R}^{n}\right)}<\infty\right\},
$$

$$
\|f\|_{\mathcal{M} \dot{K}_{p, q}^{\alpha, \lambda}\left(\mathbb{R}^{n}\right)}=\sup _{L \in \mathbb{Z}} 2^{-L \lambda}\left(\sum_{k=-\infty}^{L} 2^{k \alpha p}\left\|f \chi_{k}\right\|_{L^{q}\left(\mathbb{R}^{n}\right)}^{p}\right)^{\frac{1}{p}}
$$

with $B_{k}=B\left(0,2^{k}\right)=\left\{x \in \mathbb{R}^{n}:|x| \leq 2^{k}\right\}, A_{k}=B_{k} / B_{k-1}$ for $k \in \mathbb{Z}$ and $\chi_{k}=\chi_{A_{k}}$ for $k \in \mathbb{Z}$ be the characteristic function of the set $A_{k}$.

$\mathrm{Lu}$ and $\mathrm{Xu}$ also defined the homogeneous weak Herz-Morrey spaces. For $\alpha \in \mathbb{R}$, let $0<p \leq \infty, \lambda \geq 0$ and $0<q<\infty$, the homogeneous weak Herz-Morrey spaces $\left(W \mathcal{M} \dot{K}_{p, q}^{\alpha, \lambda}\left(\mathbb{R}^{n}\right)\right)$ is a set of measurable $f \in L_{\text {loc }}^{q}\left(\mathbb{R}^{n} /\{0\}\right)$ which completed by norm such that

$$
\|f\|_{W \mathcal{M} \dot{K}_{p, q}^{\alpha, \lambda}\left(\mathbb{R}^{n}\right)}=\sup _{\gamma>0} \gamma \sup _{L \in \mathbb{Z}} 2^{-L \lambda}\left(\sum_{k=-\infty}^{L} 2^{k \alpha p} m_{k}(\gamma, f)^{\frac{p}{q}}\right)^{\frac{1}{p}}<\infty,
$$

where $m_{k}(\gamma, f)=\left|\left\{x \in A_{k}:|f(x)|>\gamma\right\}\right|$.

Some authors have studied those spaces in different term of discussion (see [7], [8], [9], [10]). Meanwhile, in this article, the authors would like to discuss the inclusion properties and inclusion relation of the homogeneous Herz-Morrey spaces and the homogeneous weak Herz-Morrey spaces. 


\section{RESULTS AND DISCUSSION}

Now, we formulate our main results of this paper as follows:

Theorem 1.1. Let $1 \leq p_{1} \leq p_{2}<q<\infty$, then the following inclusion holds:

$$
\mathcal{M} \dot{K}_{p_{2}, q}^{\alpha, \lambda}\left(R^{n}\right) \subseteq \mathcal{M} \dot{K}_{p_{1}, q}^{\alpha, \lambda}\left(R^{n}\right) .
$$

Generally, by Theorem 1.1, we have the following inclusions of the homogeneous Herz-Morrey spaces.

Theorem 1.2. Let $1 \leq p_{1} \leq p_{2}<q<\infty$, then the following inclusion holds:

$$
L^{q}\left(R^{n}\right)=\mathcal{M} \dot{K}_{q, q}^{\alpha, \lambda}\left(R^{n}\right) \subseteq \mathcal{M} \dot{K}_{p_{2}, q}^{\alpha, \lambda}\left(R^{n}\right) \subseteq \mathcal{M} \dot{K}_{p_{1}, q}^{\alpha, \lambda}\left(R^{n}\right)
$$

Besides, we have the inclusion property of the homogeneous weak Herz-Morrey spaces, also the inclusion relation of the homogeneous Herz-Morrey spaces.

Theorem 1.3. Let $1 \leq p_{1} \leq p_{2} \leq q<\infty$, the following inclusion holds:

$$
W \mathcal{M} \dot{K}_{p_{2}, q}^{\alpha, \lambda}\left(\mathbb{R}^{n}\right) \subseteq W \mathcal{M} \dot{K}_{p_{1}, q}^{\alpha, \lambda}\left(\mathbb{R}^{n}\right) \text {. }
$$

Theorem 1.4. Let $1 \leq p \leq q$. Then the inclusion $\mathcal{M} \dot{K}_{p, q}^{\alpha, \lambda}\left(\mathbb{R}^{n}\right) \subseteq W \mathcal{M} \dot{K}_{p, q}^{\alpha, \lambda}\left(R^{n}\right)$ is proper.

The proof of each theorem will be described in the following section.

\section{THE PROOF OF THEOREM 1.1.}

For proofing Theorem 1.1., we shall show that $\|f\|_{\mathcal{M}_{\dot{K}_{p_{1}, q}^{\alpha, \lambda}\left(R^{n}\right)}^{\alpha,}} \leq\|f\|_{\mathcal{M}_{\dot{K}_{p_{2}, q}^{\alpha, \lambda}}^{\alpha, \lambda}\left(R^{n}\right)}$ by applying Hölder inequality.

Proof of Theorem 1.1. Let we first take for any $f \in \mathcal{M} \dot{K}_{p_{1}, q}^{\alpha, \lambda}\left(R^{n}\right)$, then by using Hölder inequality and $p_{1} \leq p_{2}$ we obtain that

$$
\begin{aligned}
& \|f\|_{\mathcal{M} \dot{K}_{p_{1}, q}^{\alpha, \lambda}\left(R^{n}\right)}=\sup _{L \in Z} 2^{-L \lambda}\left(\sum_{k=-\infty}^{L} 2^{k \alpha p_{1}}\left\|f \chi_{k}\right\|_{L^{q}\left(\mathbb{R}^{n}\right)}^{p_{1}}\right)^{\frac{1}{p_{1}}} \\
& \leq \sup _{L \in Z} 2^{-L \lambda}\left(\left(\sum_{k=-\infty}^{L}\left(2^{k \alpha p_{1}}\right)^{\frac{p_{2}}{p_{1}}}\right)^{\frac{p_{1}}{p_{2}}}\left(\sum_{k=-\infty}^{L}\left(\left\|f \chi_{k}\right\|_{L^{q}\left(\mathbb{R}^{n}\right)}^{p_{1}}\right)^{\frac{p_{2}}{p_{2}-p_{1}}}\right)^{1-\frac{p_{1}}{p_{2}}}\right)^{\frac{1}{p_{1}}} \\
& \leq \sup _{L \in Z} 2^{-L \lambda}\left(\left(\sum_{k=-\infty}^{L} 2^{k \alpha p_{2}}\right)^{\frac{p_{1}}{p_{2}}}\left(\sum_{k=-\infty}^{L}\left\|f \chi_{k}\right\|_{L^{q}\left(\mathbb{R}^{n}\right)}^{\frac{p_{1} p_{2}}{p_{2}-p_{1}}}\right)^{1-\frac{p_{1}}{p_{2}}}\right)^{\frac{1}{p_{1}}} \\
& \leq \sup _{L \in Z} 2^{-L \lambda}\left(\sum_{k=-\infty}^{L} 2^{k \alpha p_{2}}\left(\sum_{k=-\infty}^{L}\left\|f \chi_{k}\right\|_{L^{q}\left(\mathbb{R}^{n}\right)}^{\frac{p_{1} p_{2}}{p_{2}-p_{1}}}\right)^{\frac{p_{2}-p_{1}}{p_{1}}}\right)^{\frac{1}{p_{2}}} \\
& \leq \sup _{L \in Z} 2^{-L \lambda}\left(\sum_{k=-\infty}^{L} 2^{k \alpha p_{2}}\left\|f \chi_{k}\right\|_{L^{q}\left(\mathbb{R}^{n}\right)}^{p_{2}}\right)^{\frac{1}{p_{2}}} \\
& \leq\|f\|_{\mathcal{M} \dot{K}_{p_{2}, q}^{\alpha, \lambda}\left(R^{n}\right)} \text {. }
\end{aligned}
$$

By this observation, we know that $f \in \mathcal{M} \dot{K}_{p_{2}, q}^{\alpha, \lambda}\left(R^{n}\right)$. Hence it concludes that $\mathcal{M} \dot{K}_{p_{2}, q}^{\alpha, \lambda}\left(R^{n}\right) \subseteq \mathcal{M} \dot{K}_{p_{1}, q}^{\alpha, \lambda}\left(R^{n}\right)$. 


\section{THE PROOF OF THEOREM 1.2.}

Since it has been stated in Theorem 1.1 that $\mathcal{M} \dot{K}_{p_{2}, q}^{\alpha, \lambda}\left(R^{n}\right) \subseteq \mathcal{M} \dot{K}_{p_{1}, q}^{\alpha, \lambda}\left(R^{n}\right)$, therefore in proving Theorem 1.2, we need to prove that $L^{q}\left(R^{n}\right)=\mathcal{M} \dot{K}_{q, q}^{\alpha, \lambda}\left(R^{n}\right) \subseteq \mathcal{M} \dot{K}_{p_{2}, q}^{\alpha, \lambda}\left(R^{n}\right)$. Proof of Theorem 1.2. To prove that $L^{q}\left(R^{n}\right)=\mathcal{M} \dot{K}_{q, q}^{\alpha, \lambda}\left(R^{n}\right)$, we need to show that $\|f\|_{L^{q}\left(\boldsymbol{R}^{n}\right)}=\|f\|_{M \dot{K}_{q, q}^{\alpha, \lambda}\left(R^{n}\right)}$. Let take for any $f \in M \dot{K}_{q, q}^{\alpha, \lambda}\left(R^{n}\right)$, by applying Hölder inequality for the norm. We obtain that

$$
\begin{aligned}
\|f\|_{M \dot{K}_{q, q}^{\alpha, \lambda}\left(R^{n}\right)} & \leq \sup _{L \in Z} 2^{-L \lambda}\left(\sum_{k=-\infty}^{L} 2^{k \alpha q}\left(\left(\int_{B\left(0,2^{k}\right)}|f(x)|^{q} d y\right)^{\frac{1}{q}}\left(\int_{B\left(0,2^{k}\right)}\left|\chi_{k}\right|^{q} d y\right)^{\frac{1}{q}}\right)^{q}\right)^{\frac{1}{q}} \\
& \leq \sup _{L \in Z} 2^{-L \lambda} \sum_{k=-\infty}^{L} 2^{k \alpha}\left(\int_{B\left(0,2^{k}\right)}|f(x)|^{q} d y\right)^{\frac{1}{q}}\left(2^{k d}\right)^{\frac{1}{q}} \\
& \leq \sup _{L \in Z} 2^{-L \lambda} \sum_{k=-\infty}^{L} 2^{k \alpha+\frac{k d}{q}}\left(\int_{B\left(0,2^{k}\right)}|f(x)|^{q} d y\right)^{\frac{1}{q}} \\
& \leq C\left(\int_{B\left(0,2^{k}\right)}|f(x)|^{q} d y\right)^{\frac{1}{q}} \\
& \leq\|f\|_{L^{q}\left(R^{n}\right)}
\end{aligned}
$$

it means that $f \in L^{q}\left(R^{n}\right)$. Then $L^{q}\left(R^{n}\right) \subseteq \mathcal{M} \dot{K}_{q, q}^{\alpha, \lambda}\left(R^{n}\right)$. Meanwhile, for any $f \in L^{q}\left(R^{n}\right)$, we can find any constant $C$ such that $C=\sup _{L \in Z} 2^{-L \lambda} \sum_{k=-\infty}^{L} 2^{k \alpha+\frac{k d}{q}}$, then it shows that $f \in$ $\mathcal{M} \dot{K}_{q, q}^{\alpha, \lambda}\left(R^{n}\right)$ which means $\mathcal{M} \dot{K}_{q, q}^{\alpha, \lambda}\left(R^{n}\right) \subseteq L^{q}\left(R^{n}\right)$. Hence, it concludes that $L^{q}\left(R^{n}\right)=$ $\mathcal{M} \dot{K}_{q, q}^{\alpha, \lambda}\left(R^{n}\right)$.

Next, we have to prove that $\mathcal{M} \dot{K}_{q, q}^{\alpha, \lambda}\left(R^{n}\right) \subseteq \mathcal{M} \dot{K}_{p_{2}, q}^{\alpha, \lambda}\left(R^{n}\right)$ by showing $\|f\|_{\mathcal{M}_{\dot{K}_{2}, q}^{\alpha, \lambda}\left(R^{n}\right)} \leq\|f\|_{\mathcal{M} \dot{K}_{q, q}^{\alpha, \lambda}\left(R^{n}\right)}$. By using a similar way for proving Theorem 1.1., and since $q>p_{2}$, it is clear that $\|f\|_{\mathcal{M}_{p_{p_{2}, q}^{\alpha, \lambda}}^{\alpha, \lambda}\left(R^{n}\right)} \leq\|f\|_{\mathcal{M} \dot{K}_{q, q}^{\alpha, \lambda}\left(R^{n}\right)}$. Therefore, the proof is complete.

\section{THE PROOF OF THEOREM 1.3.}

One way for proving Theorem 1.3. is showed that $\|f\|_{W \mathcal{M} \dot{K}_{p_{1}, q}^{\alpha, \lambda}\left(R^{n}\right)} \leq$ $\|f\|_{W \mathcal{M} \dot{K}_{p_{2}, q}^{\alpha, \lambda}\left(R^{n}\right)^{*}}$

Proof of Theorem 1.3. Let take for any $f \in\|f\|_{W \mathcal{M} \dot{K}_{p_{1}, q}^{\alpha, \lambda}\left(R^{n}\right)}$, then by observing the norm of $f$ we obtain that

$$
\begin{aligned}
\|f\|_{W \mathcal{M} \dot{K}_{p_{1}, q}^{\alpha, \lambda}\left(\mathbb{R}^{n}\right)} & =\sup _{\gamma>0} \gamma \sup _{L \in \mathbb{Z}} 2^{-L \lambda}\left(\sum_{k=-\infty}^{L} 2^{k \alpha p_{1}} m_{k}(\gamma, f)^{\frac{p_{1}}{q}}\right)^{\frac{1}{p_{1}}} \\
& \leq \sup _{\gamma>0} \gamma \sup _{L \in \mathbb{Z}} 2^{-L \lambda}\left(\sum_{k=-\infty}^{L} 2^{k \alpha p_{2}} m_{k}(\gamma, f)^{\frac{p_{2}}{q}}\right)^{\frac{1}{p_{2}}} \\
& \leq\|f\|_{W \mathcal{M} \dot{K}_{p_{2}, q}^{\alpha, \lambda}\left(R^{n}\right)^{.}}
\end{aligned}
$$

By the observation, it concludes that $W \mathcal{M} \dot{K}_{p_{2}, q}^{\alpha, \lambda}\left(\mathbb{R}^{n}\right) \subseteq W \mathcal{M} \dot{K}_{p_{1}, q}^{\alpha, \lambda}\left(\mathbb{R}^{n}\right)$. 


\section{THE PROOF OF THEOREM 1.4.}

Proving Theorem 1.4 is used a similar idea as previous theorems which shall show that $\|f\|_{W \mathcal{M} \dot{K}_{p, q}^{\alpha, \lambda}\left(\mathbb{R}^{n}\right)} \leq\|f\|_{\mathcal{M} \dot{K}_{p, q}^{\alpha, \lambda}\left(\mathbb{R}^{n}\right)^{*}}$.

Proof of Theorem 1.4. Let $f \in \mathcal{M} \dot{K}_{p, q}^{\alpha, \lambda}\left(\mathbb{R}^{n}\right), a \in \mathbb{R}^{n}$, and $\gamma>0$. We observe that

$$
\left|\left\{x \in A_{k}:|f(x)|>\gamma\right\}\right|^{\frac{p}{q}} \leq\left(\int_{B\left(0,2^{k}\right)}\left|f(x) \chi_{k}\right|^{q} d x\right)^{\frac{p}{q}}=\left\|f \chi_{k}\right\|_{L^{q}\left(\mathbb{R}^{n}\right)}^{p}
$$

Multiplying both side by $\sum_{k=-\infty}^{L} 2^{k \alpha p}$, then we obtain that

$$
\sum_{k=-\infty}^{L} 2^{k \alpha p}\left|\left\{x \in A_{k}:|f(x)|>\gamma\right\}\right|^{\frac{p}{q}} \leq \sum_{k=-\infty}^{L} 2^{k \alpha p}\left\|f \chi_{k}\right\|_{L^{q}\left(\mathbb{R}^{n}\right)}^{p} .
$$

It says merely that $\|f\|_{W \mathcal{M} \dot{K}_{p, q}^{\alpha, \lambda}\left(\mathbb{R}^{n}\right)} \leq\|f\|_{\mathcal{M} \dot{K}_{p, q}^{\alpha, \lambda}\left(\mathbb{R}^{n}\right)}$, therefore $f \in W \mathcal{M} \dot{K}_{p, q}^{\alpha, \lambda}\left(\mathbb{R}^{n}\right)$. Hence, it is proved that $\mathcal{M} \dot{K}_{p, q}^{\alpha, \lambda}\left(\mathbb{R}^{n}\right) \subseteq W \mathcal{M} \dot{K}_{p, q}^{\alpha, \lambda}\left(R^{n}\right)$.

\section{CONCLUSIONS}

By this result, the author can conclude that the homogeneous Herz-Morrey spaces have inclusion properties as stated above. This result will be useful to be used in proving fractional integral on the homogeneous Herz-Morrey spaces.

\section{ACKNOWLEDGEMET}

This article research is partially supported by UIN Maliki Malang Research and Innovation Program 2020.

\section{REFERENCES}

[1] H. Gunawan, D. I. Hakim, K. M. Limanta and A. A. Masta, "Inclusion property of generalized Morrey spaces," Math. Nachr., pp. 1-9, 2016.

[2] H. Gunawan, D. I. Hakim and M. Idris, "Proper inclusions of Morrey spaces," Glasnik Mathematics, vol. 53, no. 1, 2017.

[3] H. Gunawan, D. I. Hakim, E. Nakai and Y. Sawano, "On inclusion relation between weak Morrey spaces and Morrey spaces," Nonlinear Analysis, vol. 168, pp. 27-31, 2018.

[4] H. Gunawan, E. Kikianty and C. Schwanke, "Discrete Morrey spaces and their inclusion properties," Math. Nachr., pp. 1-14, 2017.

[5] A. A. Masta, H. Gunawan and W. Setya-Budhi, "An Inclusion Property of OrliczMorrey Spaces," J. Phys.: Conf. Ser, vol. 893, pp. 1-7, 2017.

[6] C. B. Morrey, "On The Solution of Quasi-Linear Elliptic Partial Differential Equation," Transaction of The American Mathematical Society, vol. 43, no. 1, pp. 126-166, 1938.

[7] S. Lu and L. Xu, "Boundedness of Rough Singular Integral Operators on The Homogeneous Morrey-Herz Spaces," Hokkaido Math. Journal, vol. 34, pp. 299-314, 2005.

[8] M. Izuki, "Fractional Integral on Herz-Morrey spaces with variable exponent," Hiroshima Math. J., vol. 40, pp. 343-355, 2010. 
[9] Y. Shi, X. Tao and T. Zheng, "Multilinear Riesz potential on Morrey-Herz spaces with non-doubling measure," Journal of Inequality and Applications, vol. 10, 2010.

[10] Y. Mizuta and T. Ohno, "Herz-Morrey spaces of variable exponent, Riesz potential operator and duality," Complex Variable and Elliptic Equations, vol. 60, no. 2, pp. 211240, 2015. 